\title{
Extracellular heat shock proteins (eHSPs) pilot exogenous antigen into cross-presentation pathway: A superguide from extracellular world to intracellular tour
}

\author{
Yasuaki Tamura, Goro Kutomi, Jun Oura, Toshihiko Torigoe, Noriyuki Sato \\ Department of Pathlogy, Sapporo Medical University, School of Medicine
}

\begin{abstract}
Heat shock proteins (HSPs) are intracellular chaperone, some of which function as immune adjuvants as well as danger signals for immune system when released into extracellular milieu. These HSPs, along with their client polypeptides, are specifically bound by receptors on antigen presenting cells (APCs). This leads to APC differentiation along with delivery of the chaperoned peptides for cross-presentation to T cells. HSP-APC interactions occur through several receptors that mediate endocytosis or signal transduction. Most importantly, HSP associated antigens are forced to enter the cross-presentation pathway by APCs, resulting in $\mathrm{CD}^{+} \mathrm{T}$ cell activation. These unique features of HSPs for the generation of immune response will be discussed.
\end{abstract}

Keywords: Heat shock protein, Hsp90, Cross-presentation, Antigen presenting cell, MHC class I molecule

(Received August 13, 2007; Accepted August 31, 2007)

\section{Introduction: HSP-mediated cross-presentation by antigen-presenting cells}

It has been well demonstrated that immunization with tumor-derived HSPs or HSP complexed with an antigen peptide elicits tumor- or antigen-specific $\mathrm{CD}^{+} \mathrm{T}$ cell responses. Above all, Hsp70- and gp96-antigen complexes are well-studied and have been shown to be immunogenic and potent in stimulating the generation of tumorspecific CTLs. Hsp70- and gp96-based vaccines have been tested in early-phase clinical trials in solid tumors as well as in lymphoma and leukemias; all showed minimal toxicity and potential efficacy ${ }^{1-3)}$. Phase III clinical trials using tumor-derived Hsp70 and gp96 as vaccines are ongoing for melanoma and renal cell carcinoma.

The ability of HSPs to facilitate the cross-presentation of MHC class I-restricted epitopes and to prime $\mathrm{CD}^{+} \mathrm{T}$ cell effector responses is well established ${ }^{4,5}$. Although, immunized HSPs are exogenous antigens, these HSPantigen complexes can gain access to the class I antigen presentation pathway, resulting in cross-presentation. The immune response has been attributed to the ability of HSPs to form stable complexes with tumor-derived antigenic peptides, thereby facilitating the cross-presentation of MHC class I-restricted epitopes and priming of $\mathrm{CD} 8^{+}$

Correspondence to: Yasuaki Tamura, Department of Pathlogy, Sapporo Medical University, School of Medicine
$\mathrm{T}$ cell responses.

Dendritic cells (DCs) are main conductor for efficient cross-presentation. Recent reports have shown that antigen-presenting cells (APCs) such as dendritic cells can internalize HSPs by receptor-mediated endocytosis and direct chaperoned proteins/peptides into the intracellular pathway for MHC class I-restricted presentation to $\mathrm{CD} 8^{+}$ $\mathrm{T}$ cells, concomitant with the induction of dendritic cell maturation and cytokine secretion. In fact, some HSP receptors expressed on APCs have recently been identified. $\mathrm{CD}{ }^{6)}, \mathrm{LOX}^{6} 1^{7)}, \mathrm{CD} 40^{8)}$ and SR-A ${ }^{9)}$ have been proved to be common receptors for HSPs. However, the underlying mechanism for efficient cross-presentation, in particular, how the HSP-antigen complex can enter the MHC class I pathway, remains unclear.

Furthermore, recent studies have shown that HSPpeptide complexes can also lead to antigen presentation on MHC class II molecules, thus activating $\mathrm{CD}^{+} \mathrm{T}$ cells ${ }^{10,11)}$. Therefore, it is possible that uptake of HSPpeptides complexes leads to antigen presentation on both MHC class I and class II molecules on dendritic cells, thus activating $\mathrm{CD} 8^{+} \mathrm{CTL}$ as well as $\mathrm{CD} 4^{+} \mathrm{T}$ cells. However, Shild et al. have reported that, although antigen peptides chaperoned by gp96 can be presented in the context of both MHC class I and class II molecules, immunization with gp96 elicits CD8-biased T cell respons$\mathrm{es}^{12)}$. Doody et al have also demonstrated same results ${ }^{5)}$. Therefore, it is essential to know the effects of the HSPantigen complex on tumor antigen presentation via class 
I and class II pathways in vivo because such knowledge is crucial for the development of effective HSP-based immunotherapies, especially in the case of protein antigens in association with HSPs.

In contrast to Hsp70 and gp96, the role of Hsp90 in cross-presentation remains unclear. Hsp90 is the most abundant protein in the cytoplasm; therefore it is assumed that Hsp90 also plays an important role in crosspresentation. However, it is not clear whether Hsp90 is involved in tumor immunity. Udono reported, for the first time, that immunization with Hsp90 purified from tumor elicited tumor-specific CTL responses ${ }^{13)}$. Very recently, Kunisawa and Shastri reported that Hsp90 chaperoned C-terminal flanking antigenic peptides ${ }^{14)}$. These results have led to much interest in the importance of Hsp90 in antigen presentation. Taking these facts into consideration, it is conceivable that Hsp90 does participate in antigen presentation and possibly cross-presentation. In this paper, we will discuss the mechanism of HSPmediated cross-presentation and the players involved in this intriguing immune response.

\section{HSPs act as danger signals to the immune system through HSP receptors expressed on APCs.}

It is suggested that extracellular HSPs (eHSPs) act as "danger signals" to the immune system in the case of life-threatening events (Fig. 1). When viral infection or tumor cell damage occurs, cell-associated antigens, such as HSPs-antigen complexes are released into the extracellular milieu, APCs such as dendritic cells and macrophages, detect the signals through certain receptors, resulting in intracellular signaling ${ }^{15)}$. HSP receptors are divided into 2 categories, one is toll-like receptor (TLR) for mainly signaling for DC maturation and activation, and the other is endocytic receptors for cross-presentation. TLR-2 has been shown to be the receptor for Hsp70 ${ }^{16)}$. TLR-4 is the receptor for Hsp70 ${ }^{16,17)}$. However, doubts were raised as to what extent this effect was due to lipopolysaccharide contaminations of the HSP preparations. It is required re-examination for this phenomenon using HSPs, nominally endotoxin-free. In contrast, HSP-specific endocytic receptors expressed on the APCs, including CD91 for gp96, Hsp70 and Hsp90 6,18), SR-A for gp96 and calreticulin ${ }^{9)}$, LOX-1 for Hsp70 ${ }^{7)}$, and CD40 for $\mathrm{Hsp} 70^{19)}$, were identified. However, it is still unknown how HSP-antigen complexes are transported and where HSP releases chaperoned antigens. What is the fate of HSPs after endocytosis? What actors are responsible for translocating the antigen from the endosome to cytosol? Additional studies will be required to understand these unsolved issues.

\section{The roads to MHC class I presentation}

MHC class I molecules principally present peptides derived from endogenous protein to cytotoxic $\mathrm{T}$ cells. However, in certain antigen-presenting cells, peptides derived from exogenous antigens are also presented by MHC class I molecules. At least four independent pathways of protein processing and subsequent peptide presentation by class I molecules have been described. The dominant pathway uses endogenously synthesized proteins that have been processed in the cytosol by proteasomes. Peptides are transferred by transporterassociated antigen processing (TAP) to the ER where they bind in the grooves of nascent MHC class I molecules. The peptide/class I MHC heavy chain $/ \beta 2 \mathrm{~m}$ complex is then transported via the Golgi apparatus to the cell surface. In second and third pathways, exogenous antigens are internalized and processed into peptides that are transported to the ER to bind MHC class I. One is the cytosolic leak of internalized antigens by phagocytosis, macropinocytosis, and enocytosis, resulting in degradation by proteasomes ${ }^{20,21)}$. Degraded peptides are then transported through TAP into the ER. However, the mechanism for the translocation of exogenous antigens to cytosol and players involved in this translocation remain unknown. Another pathway is via ER-phagosome fusion. When exogenous antigens are engulfed into phagosomes, the phagosomal membrane and ER membrane fuse with each other, forming ER-phagosome compartments $^{22,23)}$. These ER-phagosome fusion compartments involve TAP molecules and proteasomes outside of the membrane ${ }^{24)}$. Phagocytosed antigens are pumped out the ER-phagosome fusion compartment through sec61. This is called the ER-associated degradation (ERAD) mechanism. Then proteasomes, which are attached to the outer face of the membrane of ER-phagosome fusion, degrade antigens into peptides, followed by entry into the ER again through TAP molecules, and the resulting antigen peptides bind to MHC class I molecules ${ }^{25,26)}$. In contrast, at least some exogenous peptides or proteins appear to reach MHC class I through a pathway completely independent of the ER. In this fourth pathway, endosomal processing and endocytosed class I MHC molecules may be involved. MHC class I molecules have been shown to internalize from the cell surface in T cells, B cells, fibroblasts, and macrophages. Recycling of endocytosed class I MHC molecules back to the cell surface has also been observed $^{27)}$. The endocytosis and recycling of class I MHC may facilitate peptide exchange, allowing class I MHC molecules to bind multiple peptides in one lifetime. Antigen presentation mediated by the latter three types mentioned above is called cross-presentation, allows display of exogenous antigens in the context of MHC class I molecules. This is particularly important in 


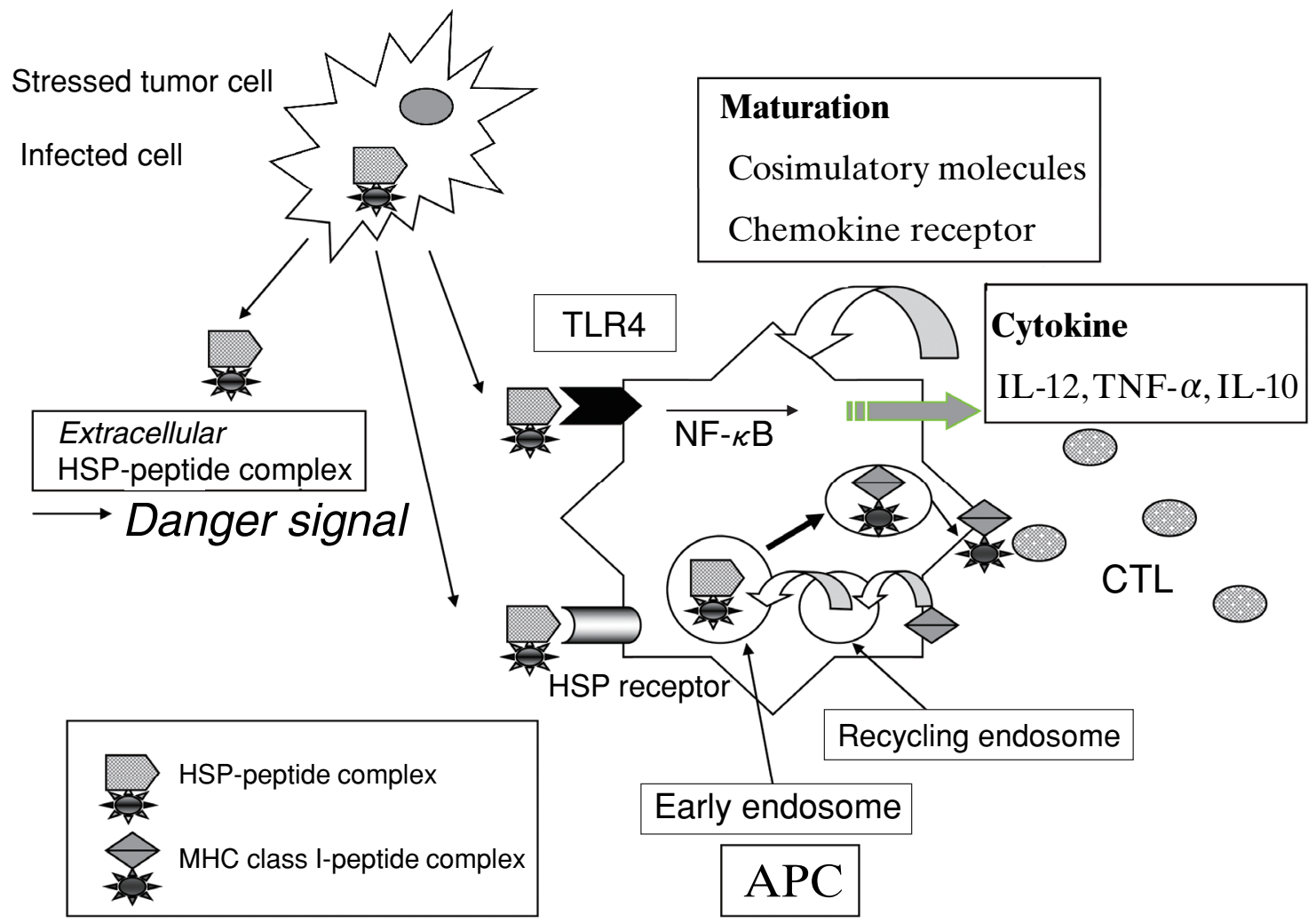

Fig. 1 Role of extracellular HSP-peptide complex as a danger signal. HSP-peptide complexes are acquired by bone marrow-derived antigen presenting cells (APC) and are cross-presented to cytotoxic T lymphocytes (CTL). HSPpeptide complexes bind to Toll-like receptor (TLR) 4 and induce maturation and activation of dendritic cells (DC). On the other hand, HSP-peptide complexes also bind to HSP receptor, such as CD91, LOX-1, SR-A, on the DC, followed by endocytosis. Internalized HSP-peptide complexes are shuttled into MHC class I pathway via endosomal pathway, and induce CTL response. HSP-peptide complexes elicit both innate and adaptive immunity simultaneously, indicating that HSP-peptide complexes act as danger signal.

host defense against infectious diseases and cancers that cannot access the classical pathway for MHC class I presentation. Moreover, for the development of the efficient cancer vaccine, it is necessary to establish the methods for delivering exogenous cancer vaccine into cross-presentation pathway.

Internalization of exogenous antigens may allow cell fragments, intracellular pathogens and proteins to be degraded in the endocytic pathway by mechanisms involving reduction, unfolding and lysosomal proteolysis. Such a process could contribute to cross-presentation by facilitating the exchange of previously loaded MHC class I-associated peptides for newly generated peptides derived from exogenous proteins. Cycloheximide treatment, which blocks the biosynthetic pathway of MHC I, indicated that the class I present in the endocytic compartments was derived from the cell surface. We have confirmed the presence of MHC class I in the endocytic compartments of murine bone marrow-derived DCs. Furthermore, as described recently, we have demonstrated that co-localization of the receptor-mediated endocytosed exogenous Hsp70/90-antigen complex with
MHC class I in the early endosomes of the DCs. These observations suggest that antigens derived from the exogenous Hsp70/90 may be loaded onto recycling MHC class I, after which the MHC class I/peptide complex is transported to the cell surface.

\section{Impact on HSP-antigen complex in a cross- presentation pathway}

Considering the significance of the HSP-peptide complex in cross-presentation, this model suggests that a 'pre-processed antigen' would be required as it would be inefficient for a whole protein to be degraded nonspecifically within the endocytic compartments by resident catabolic enzymes. Not only would this be a slow process, it would be by chance alone that an appropriate peptide capable of binding the MHC class I groove would be generated. The notion of such preprocessed antigens fits well with a role for HSPs as chaperones for peptide antigens. Proteins synthesized within the cell would be processed within the endogenous class I antigen presentation pathway leading to the generation of 
HSP-peptide complexes. These complexes are ideal chaperones of antigenic peptides for the transfer of antigens to DCs for a number of reasons. Once generated within the cell, the HSP-peptide complex might be released into the extracellular milieu during cell necrosis because of viral infection and intervention of cancer, resulting in taking-up by the immature DC and acting as a danger signal. At the same time, antigenic peptides chaperoned by HSPs are efficiently presented in the context of MHC class I molecules and immediately activate the host's immune responses. As described earlier, Hsp90 binds precursor (pre-processed) peptides generated in the cells and thus, endosomal processing is a suitable mechanism for pre-processed peptides. HSP would also serve to protect the peptide antigen from degradation upon entry into the endocytic compartment of the DC. In addition, an as yet uncharacterized lysosomal enzyme may play a role in the processing of internalized antigens for generation of MHC I epitopes. Recently, Rock et al. have demonstrated that DC-restricted cathepsin S plays an important role in the processing of exogenous antigens for the generation of MHC I antigenic determinants in the early endosome ${ }^{28)}$.

\section{Significance of cross-presentation in vivo: impact on epitope generation via endosomal processing and proteasomal processing}

Although DCs are capable of using an endocytic exchange mechanism to create MHC class I-peptide complexes, typical somatic cells have only the classical pathway for the generation of MHC class I-presenting peptides. For $\mathrm{CD}^{+} \mathrm{T}$ cells induced by cross-presentation to be functionally useful against pathogen-infected cells, it would seem that the epitope generation mechanism used should be the same as those in classical MHC class I processing. An endosomal exchange mechanism in which peptides are generated by different proteases in radically different conditions from those in the endogenous pathway, therefore seems unlikely to contribute substantially to the $\mathrm{CD}^{+} \mathrm{T}$ cell repertoire. Cytoplasmic processing, including proteasomal proteolysis, and ERbased trimming would be expected to be involved to generate the same peptide sequences as those made by nonhematopoietic cells. In fact, although partial proteolysis may occur in the endocytic pathway, extensive experimental evidence suggests that exogenous antigens must reach the cytoplasm to be efficiently cross-presented. In DCs, the presentation of exogenous antigens is unaffected by both chloroquine and inhibitors of lysosomal proteolysis. Exogenous antigen presentation is, however, highly sensitive to specific inhibitors of the proteasome, indicating that cytoplasmic proteolysis is the main form of epitope generation in the cross-presentation pathway. In contrast, the HSP-mediated cross-presentation pathway has been shown to involve both a proteasomal pathway and an endocytic-recycling pathway. We have demonstrated that a tumor-derived Hsp70-peptide complex is efficiently cross-presented to peptide-specific CTLs by DCs and this presentation is dependent on TAP molecules. In addition, in vitro generated Hsp70- and gp96-antigen complexes have been shown to be crosspresented via a TAP-dependent parhway ${ }^{29-31)}$. This fact suggests that processing and loading a peptide onto MHC class I requires translocation of the antigen from the endocytic compartment to the cytosolic pathway. Rodriguez et al. demonstrated that DCs have a unique membrane transport pathway linking the lumina of endocytic compartments and the cytosol ${ }^{20)}$. Thus, in DCs, the exogenous HSP-chaperoned antigen in the endocytic compartment is released into the cytosol, where it follows the classical proteasome- and TAP-dependent class I pathway for presentation. Further studies to define the precise mechanisms for Hsp70- and Gp96-chaperoned peptide trafficking may reveal a new paradigm for crosspresentation.

\section{Cross-presentation of exogenous Hsp90-peptide complex by dendritic cells}

Hsp90 is a one of the most abundant proteins within cells and is overexpressed in many cancer cells. Therefore, once cancer cells become necrotic, much Hsp90 would be released from cells and might be a danger signal, subsequently eliciting cell-specific immune responses. It has been demonstrated that the tumorderived Hsp90-peptide complex elicits tumor-specific immunity. At present, however, the processing pathway yielding the transfer of exogenous Hsp90-associated peptide antigens to MHC class I molecules is unknown.

We examined the roles of Hsp90 in MHC class I-restricted cross-presentation using bone marrowderived dendritic cells (DCs) as $\mathrm{APCs}^{32}$. First, we tested whether Hsp90-peptide complexes reconstituted in vitro were taken-up and associated peptides presented in the context of MHC class I molecules by DCs. To monitor the MHC class I antigen-processing pathway, we used Hsp90 reconstituted in vitro with the C-terminal extended version of VSV8 (RGYVYQGL), VSV-C (RGYVYQGLKSGNVSC: 15mer) to moniter the processing of the precursor peptide. The Hsp90-VSV-C peptide complex was cocultured with DCs for 2 hours, followed by incubation with a VSV8-specific CTL clone. The culture supernatant was assayed for the production of IFN- $\gamma$. VSV-C-loaded Hsp90 was processed and presented by $\mathrm{H}-2 \mathrm{~K}^{\mathrm{b}}$ and recognized by the VSV8-specific CTL clone, but not Hsp90 or VSV-C alone. In the presence of an anti-H-2 $\mathrm{K}^{\mathrm{b}} \mathrm{mAb}$ during the presentation assay, the presentation of VSV8 to the specific CTL clone was completely abolished. These data suggested that 
Hsp90-bound VSV-C peptides were processed to VSV8 within the cells with subsequently gained access to the MHC class I pathway. Intriguingly, this presentation occurred within $15 \mathrm{~min}$, indicating that very rapid and efficient processing might be achieved within DC (Fig.2A).

Next, we investigated whether the Hsp90-mediated MHC class I pathway required functional TAP molecules. To test this, we used DCs derived from the TAP1 ${ }^{-1}$ mouse. Surprisingly, DCs from the TAP1 $1^{-1}$ mouse could also process and present Hsp90-bound VSV-C peptides as efficiently as DCs from the wild-type mouse.

We also tested another well-characterized $\mathrm{H}-2 \mathrm{~K}^{\mathrm{b}}$ restricted $\mathrm{OVA}_{258-265}$ antigen system, Hsp90 reconstituted in vitro with the $\mathrm{C}$-terminal extended version of SL8 (OVA $_{258-265}$ ), SL8-C peptide (OVA $258-270: 13$ mer). The Hsp90-SL8-C peptide complex was cocultured with DCs for 2 hours, followed by incubation with SL8-specific B3Z T cell hybridoma. As shown in Fig. 3B, the Hsp90SL8C peptide complex was processed and presented by $\mathrm{H}-2 \mathrm{~K}^{\mathrm{b}}$ and recognized by $\mathrm{B} 3 \mathrm{Z} \mathrm{T}$ cell hybridoma, but not Hsp90 or SL8-C alone, in a TAP-independent manner (Fig. 2B). These experiments demonstrate that a TAPindependent pathway is used for Hsp90-mediated MHC class I presentation.

\section{Intracellular localization of endocytosed exogenous Hsp90-peptide complexes}

Using laser confocal microscopy, we found that Hsp90-peptide complexes accumulated only in the endosome and did not reach the stage of the lysosome. We also examined whether Hsp90 accumulation in the endosome was due to temperature-dependent endocytosis. As expected, at $4^{\circ} \mathrm{C}$, labeled $\mathrm{Hsp} 90$ remained on the cell surface, but internalization was evident after incubation at $37^{\circ} \mathrm{C}$ following a 10 -min internalization period. According to a competition assay, DCs express Hsp90 receptor on the cell surfaces. Identification of receptor (s) will be necessary to elucidate the mechanism responsible for cross-presentation of exogenous Hsp90-antigen complexes.

\section{The pathway for Hsp90-mediated cross- presentation}

To investigate where the Hsp90-associated antigenic peptides bind to MHC class I molecules, we stained $\mathrm{H}-2 \mathrm{~K}^{\mathrm{b}}$ molecules and exogenous Hsp90-peptide complexes. After $20 \mathrm{~min}$ of endocytosis, Alexa-labeled Hsp90 colocalized with endocytosed $\mathrm{H}-2 \mathrm{~K}^{\mathrm{b}}$ molecules. The results showed the internalization and co-localization of $\mathrm{H}-2 \mathrm{~K}^{\mathrm{b}}$ and Hsp90 was evident in the early endosome. This finding suggested that HSP-bound peptides might be transferred to MHC class I molecules in the endosome, where recycled MHC class I molecules from the plasma mem-

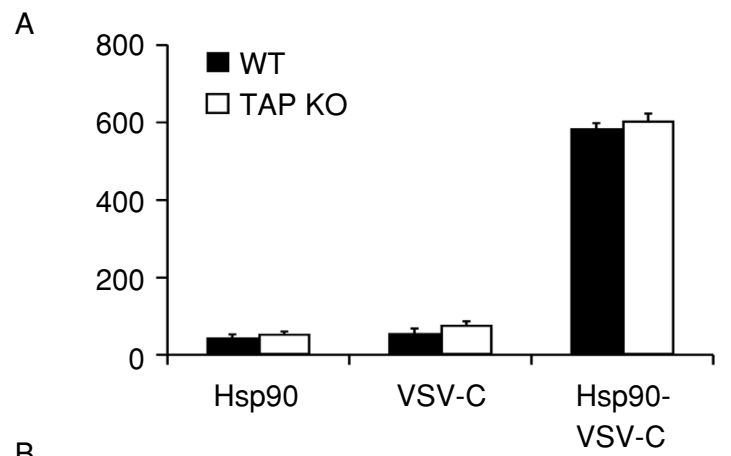

B

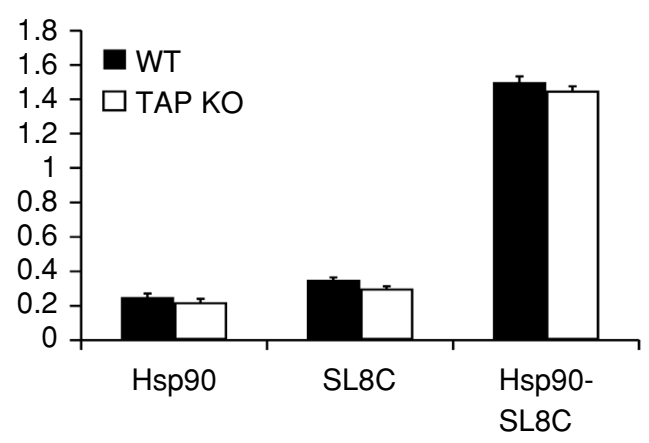

Fig. 2 Precursor peptides chaperoned by Hsp90 are cross-presented via a TAP-independent pathway. A. Hsp90-VSV-C complex, Hsp90 or VSV-C was loaded to BMDCs for $2 \mathrm{~h}$ and a VSV8-specific CTL clone was added. IFN- $y$ in the culture supernatant was measured by ELISA. BMDCs from the $\mathrm{TAP}^{-/-}$mouse could also process and present Hsp90chaperoned VSV-C peptides efficiently as compared to BMDCs from the wild type mouse. B. SL8 precursor peptide, SL8C was also processed and crosspresented by BMDCs via a TAP-independent pathway.

brane are available. The peptide-MHC class I complexes generated in the endosome are then transported to the cell surfaces of the DCs, where specific CTLs recognize them.

Recycling of endocytosed MHC class I molecules back to the cell surface has been observed. Some of the recycled MHC class I molecules can be loaded into endosomes with peptides derived from endocytosed molecules $^{27)}$. Therefore, to confirm whether this presentation really utilizes recycled MHC class I molecules, we treated DCs with primaquine, which blocks the membrane recycling pathway. DCs incubated in the presence of this drug could not present the Hsp90-chaperoned SL8Cderived SL8 peptide. This result indicated that precursor peptides chaperoned by Hsp90 or processed peptides entered into recycling endosomes and transferred onto recycling MHC class I molecules, which returned to the cell surface and stimulated B3Z T cell hybridoma. To analyze the involvement of vacuolar acidification of endosomal compartments, DCs were incubated with Hsp90-SL8C in the presence of chloroquine, a known inhibitor of acidification of endosomal compartments. 


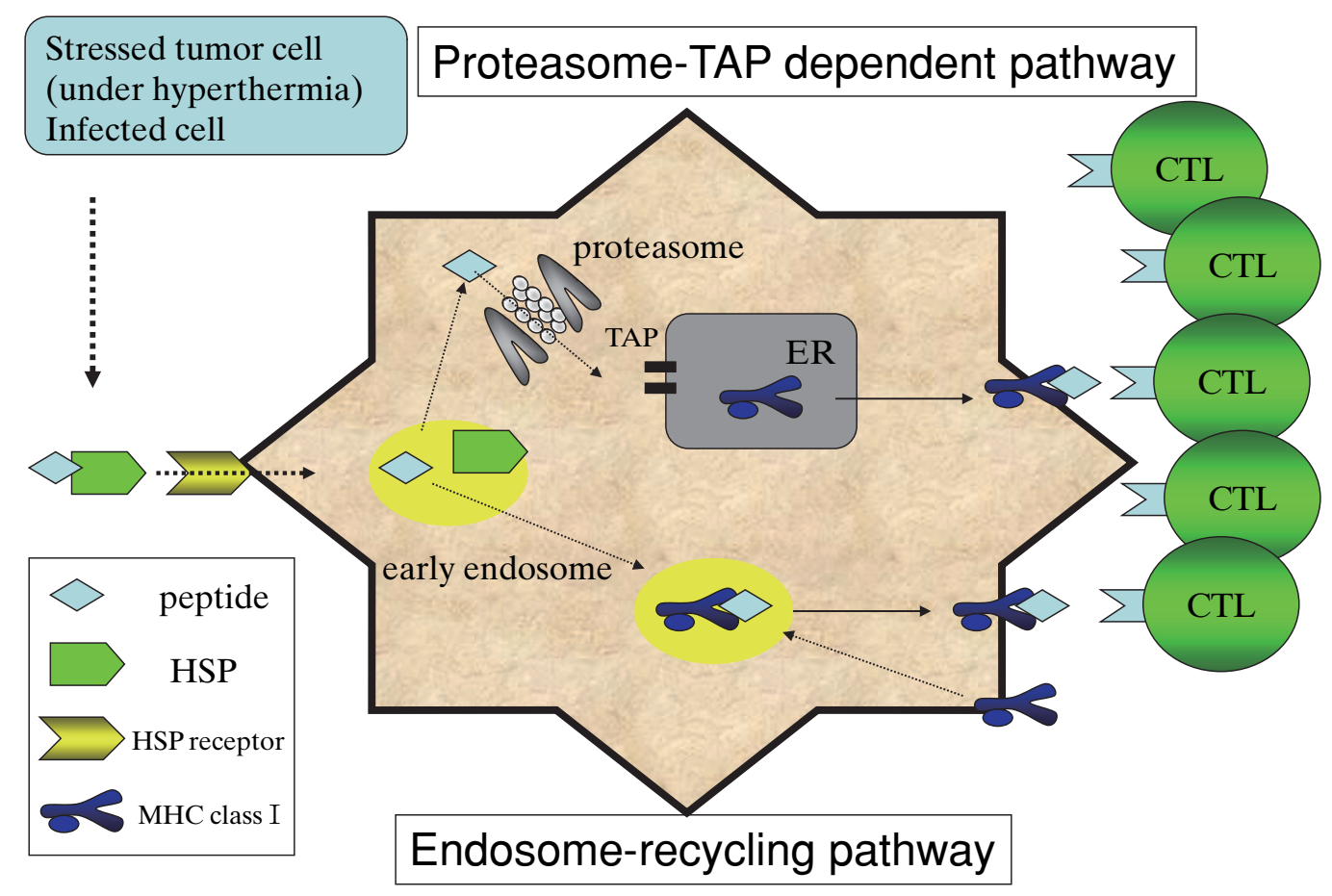

Fig. 3 Possible pathway of Hsp90-antigen complex-mediated cross-presentation. Internalized Hsp90-antigen complexes through receptor-mediated endocytosis follow 2 distinct MHC class I pathways. (1) Internalized Hsp90antigen complexes are transferred to the cytosol and imported into the ER in a TAP-dependent fashion. Hsp90chaperoned antigens are degraded in the cytosol by proteasome and further trimmed by cytosolic peptidases. The resulting peptides are transported into the lumen of the ER for loading on newly synthesized MHC class I molecules. (2) Alternatively, internalized antigens chaperoned by Hsp90 are processed and loading in the endocytic pathway onto MHC class I molecules that are recycled from the plasma membrane, independently of TAP.

Chloroquine strongly inhibited Hsp90-mediated presentation without affecting SL8 peptide presentation. Thus, acidification of endosomal compartments is necessary for processing of Hsp90-chaperoned precursor peptides (Fig. 3).

\section{Hsp90-chaperoned precursor peptides are processed by the endosomal protease}

We used protease inhibitors to investigate the proteolytic processes involved in the Hsp90-mediated TAPindependent cross-presentation pathways. In wild-type DCs, a broadly active cysteine protease inhibitor, leupeptin, almost completely inhibited the cross-presentation of Hsp90-SL8C. In contrast, the aspartic protease inhibitor pepstatin did not affect the cross-presentation. Cathepsins S, D and L are known to be the major cysteine proteases in endocytic compartments. We therefore examined the roles of various cathepsins in this pathway. Cathepsin D- and cathepsin L-specific inhibitors did not affect the cross-presentation, whereas a cathepsin $\mathrm{S}$ inhibitor completely blocked cross-presentation. Cathepsin $\mathrm{S}$ is a cysteine protease that is preferentially expressed in APCs, including DCs, macrophages, and B cells within endocytic compartments. Therefore, our data indicate that cathepsin $\mathrm{S}$ is a critical enzyme in TAP-independent Hsp90-mediated cross-presentation on MHC class I molecules and that the presented peptides are indeed generated in endosomal compartments.

\section{Advantages of Hsp90-antigenic protein complexes}

As described above, we have shown that Hsp90chaperoned precursor peptides are efficiently processed and presented by MHC class I molecules. To extend the range of HSP-based immunotherapy, we examined whether whole protein antigens chaperoned by Hsp90 were processed and presented by MHC class I molecules as well as class II molecules. The advantages of using protein antigens for cancer immunotherapy are that they can (1) provide an inherent polyvalent vaccine for $\mathrm{CD}^{+} \mathrm{T}$ cells, and (2) they include $\mathrm{CD} 4^{+}$helper epitopes, required for efficient CTL induction and proliferation. However, protein antigens themselves are not primarily immunogenic and therefore an immunostimulatory adjuvant is necessary for effective $\mathrm{T}$ cell responses. Given the well-known ability of HSP to forms complexes with naturally synthesized proteins, it is possible that Hsp90protein antigen complexes could elicit antigen-specific 
CTL responses and Th responses as well. Therefore, we investigated the impact of Hsp90 on the presentation of exogenous protein antigens using OVA protein as a model antigen. We observed that the Hsp90-OVA antigen complex generated in vitro was very efficiently and selectively presented via the MHC class I pathway both in vitro and in vivo. Surprisingly and unexpectedly, we observed that the cross-presentation of Hsp90-OVA complexes was involved both TAP-dependent and -independent pathways, unlike the results of Hsp90-precursor peptide complex. These results provide a rationale for the development of novel vaccination strategies for cancer immunotherapy.

\section{Hsp90-OVA complex is efficiently cross- presented by DCs.}

We evaluated cross-presentation of the Hsp90-OVA protein complex. DCs were pulsed with Hsp90 alone, free OVA, a simple mixture of the two or the two in a complex generated in vitro for $2 \mathrm{hrs}$ at $37^{\circ} \mathrm{C}$, then fixed, washed and cultured with $\mathrm{B} 3 \mathrm{Z} \mathrm{CD}^{+} \mathrm{T}$ cell hybridoma. The Hsp90-OVA complex elicited strong CTL responses, whereas Hsp90 or OVA alone did not lead to CTL responses. Notably, when we pulsed the simple mixture of Hsp90 and OVA, we did not detect significant CTL responses. These results show that binding to Hsp90 is essential for cross-presentation of OVA.

\section{Hsp90-OVA complex is efficiently and preferentially presented through MHC class I, but not class II pathway}

We also tested whether the Hsp90-OVA complex was presented through the MHC class II pathway, and elicited $\mathrm{CD}^{+} \mathrm{T}$ cell responses. DC from B6C3F1 were pulsed with free OVA or Hsp90-OVA complex for $2 \mathrm{hrs}$ at $37^{\circ} \mathrm{C}$, then fixed, washed and co-cultured with $\mathrm{B} 3 \mathrm{Z}$ $\mathrm{CD}^{+} \mathrm{T}$ cell hybridoma or $\mathrm{KZO} \mathrm{CD} 4^{+} \mathrm{T}$ cell hybridoma. Stimulation with free OVA led not to CTL responses but strong $\mathrm{CD} 4^{+} \mathrm{T}$ cell responses. In contrast, stimulation with the Hsp90-OVA complex elicited significantly weaker $\mathrm{CD}^{+} \mathrm{T}$ cell responses than free OVA, whereas it induced robust CTL responses. These findings suggest that the Hsp90-OVA complex is presented much more selectively through the MHC class I pathway than the MHC class II pathway.

To examine the differences in presentation efficacy between the Hsp90-OVA complex and free OVA, a pulse-chase experiment was performed. DC were pulsed with OVA alone or the Hsp90-OVA complex at $37^{\circ} \mathrm{C}$, and harvested at different times from $10 \mathrm{~min}$ to $2 \mathrm{hrs}$, then fixed, washed and cultured with B3Z or KZO. B3Z responses were seen after 10 to $30 \mathrm{~min}$ of stimulation with the Hsp90-OVA complex, although no KZO re- sponses were detected up to $1 \mathrm{hr}$ with free OVA. These data demonstrate that cross-presentation of the Hsp90OVA complex is more rapid and efficient than presentation of free OVA.

\section{Why and how do HSP-antigen complexes skew the $\mathrm{CD8}^{+} \mathrm{T}$ cell responses but not $\mathrm{CD} 4^{+} \mathrm{T}$ cell responses?}

Ramirez et al. reported that a gp96-peptide complex elicited $\mathrm{CD}^{+} \mathrm{T}$ cell responses but not $\mathrm{CD} 4^{+} \mathrm{T}$ cell responses ${ }^{12)}$. We have also observed that presentation of exogenous Hsp90-chaperoned peptides and protein antigens tends to drive the $\mathrm{CD}^{+} \mathrm{T}$ cell response. Although, protein antigens such as OVA protein contain both a $\mathrm{CD}^{+} \mathrm{T}$ cell epitope and $\mathrm{CD} 4^{+} \mathrm{T}$ cell epitope, if OVA is chaperoned by Hsp90, the Hsp90-OVA complex is taken up by receptor-mediated endocytosis and enters the cross-presentation pathway, followed by $\mathrm{CD} 8^{+} \mathrm{T}$ cell responses. In contrast, the soluble form of OVA protein is pinocytosed and follows the classical class II pathway. As for what drives Hsp90-antigen complexes into the cross-presentation pathway, the regulators for this transport are unclear and further studies will be required to elucidate the precise mechanism.

\section{The mechanism for translocation of Hsp90- chaperoned antigens from endosomes to cytosol.}

The mechanism for antigen escaping to the cytosol remains unknown, as is whether the Hsp90-antigen complex or its components separately escape to the cytosol. It is possible that the Hsp90-antigen complex first needs to be preprocessed in the endosomal compartments before being transferred to the cytosol to be further degraded by proteasomes. One possibility is that the mildly acidic $\mathrm{pH}$ in the endocytic compartments plays an important role for the transport of ingested antigens, and another is that delayed fusion with the late endosome/lysosome is important for the transport. Immature DCs maintained mildly acidic $\mathrm{pH}$ in the endocytic compartment even after antigen uptake and could transport these antigens into the cytosol ${ }^{21)}$. Chloroquine treatment inhibits the acidification of the endocytic compartments. Our data indicated that chloroquine treatment inhibited Hsp90-OVA presentation by DCs in both TAP-dependent and -independent pathways. This suggested that antigen transport was dependent on mildly acidic pH-inducible machinery in the endocytic compartments of DCs. However, a recent report showed that treatment with chlorquine or $\mathrm{NH}_{4} \mathrm{Cl}$ enhanced the efficiency of cross-presentation ${ }^{33)}$. These treatments accelerated export of exogenous soluble antigens from endocytic compartments to cytosol, thereby enhancing cross-presentation. The difference between 


\section{The presentation pathway of Hsp90-protein complexes}

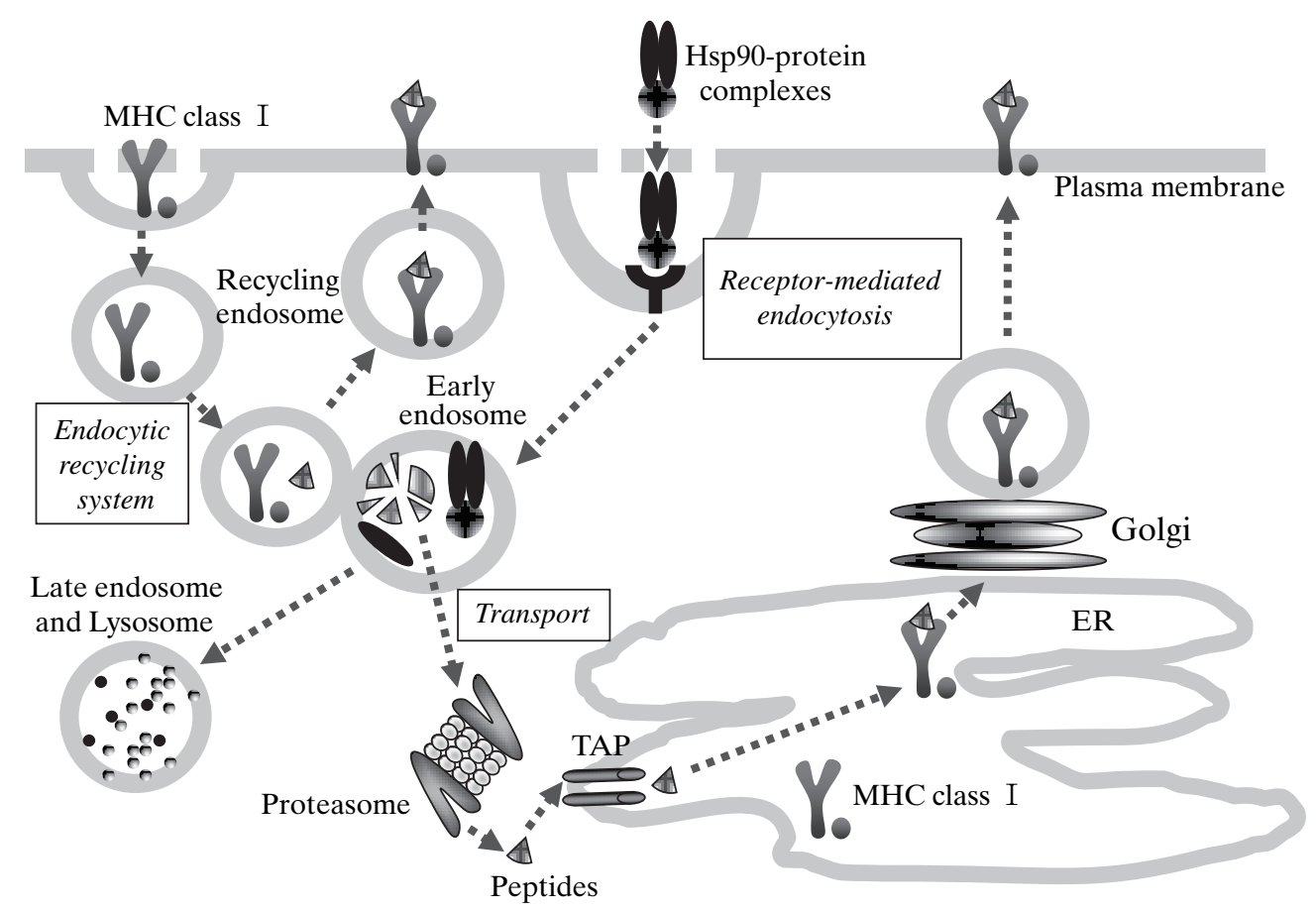

Fig. 4 Route of access to the cytosol for internalized Hsp90-antigen complexes. Pathways for generation of Hsp90chaperoned peptide-MHC class I complexes. Antigens are first cleaved endosomal protease, such as cathepsin S, and resulting peptide intermediates then enter into cytosol, followed by proteasomal degaradation. A small fraction of peptide intermediates are further trimmed by endosomal protease within endosomes, thereafter reach recycling endosomes and are loaded onto MHC class I molecules, which the return to plasma membrane occurs. It remains unclear what the mechanisms are by which they traverse the endosomal membrane or if it reflects the existence of a specific channel or a translocator.

our results and theirs was the method of uptake by DCs. In our case it occurred via receptor-mediated endocytosis, whereas they indicated that it occurred via phagocytosis or macropinocytosis. In addition, the regulators for the transport are still unclear. We are still on the road to complete understanding of HSP-mediated immune regulation, and further studies will be required to elucidate the precise mechanism (Fig. 4).

\section{Conclusion}

Although HSPs are primarily cytosolic proteins, they play an important role as a "danger signal" in the extracellular milieu on behalf of immune surveillance. Above all, Hsp90 is one of the most abundant cytosolic proteins and elicits intriguingly efficient and rapid CTL responses. In this meaning, Hsp90 is a "smart and excellent guide" for the MHC class I cross-presentation pathway. A forthcoming issue is to elucidate the mechanism of the driving force toward the $\mathrm{CD} 8^{+} \mathrm{T}$ cell response mediated by HSPs. In addition, how endocytosed HSP-chaperoned antigens are translocated to be processed? These findings will clarify the impact of HSP as a danger signal in the etiologies of autoimmune diseases and tumor immunity.

\section{References}

1) Castelli, C., L. Rivoltini, F. Rini, F. Belli, A. Testori, M. Maio, V. Mazzaferro, J. Coppa, P.K. Srivastava, and G. Parmiani. 2004. Heat shock proteins: biological functions and clinical application as personalized vaccines for human cancer. Cancer Immunol Immunother 53:227-233.

2) Mazzaferro, V., J. Coppa, M.G. Carrabba, L. Rivoltini, M. Schiavo, E. Regalia, L. Mariani, T. Camerini, A. Marchiano, S. Andreola, R. Camerini, M. Corsi, J.J. Lewis, P.K. Srivastava, and G. Parmiani. 2003. Vaccination with autologous tumor-derived heat-shock protein gp96 after liver resection for metastatic colorectal cancer. Clin Cancer Res 9:3235-3245.

3) Rivoltini, L., C. Castelli, M. Carrabba, V. Mazzaferro, L. Pilla, V. Huber, J. Coppa, G. Gallino, C. Scheibenbogen, P. Squarcina, A. Cova, R. Camerini, J.J. Lewis, P.K. Srivastava, and G. Parmiani. 2003. Human tumor-derived heat shock protein 96 mediates in vitro activation and in vivo expansion of melanoma- and colon carcinoma-specific T cells. J Immunol 171:3467-3474.

4) Delneste, Y. 2004. Scavenger receptors and heat-shock protein-mediated antigen cross-presentation. Biochem Soc Trans 32:633-635.

5) Doody, A.D., J.T. Kovalchin, M.A. Mihalyo, A.T. Hagymasi, C.G. Drake, and A.J. Adler. 2004. Glycoprotein 96 can chaperone both MHC class I- and class II-restricted epitopes for in vivo presentation, but selectively primes $\mathrm{CD} 8^{+} \mathrm{T}$ cell effector function. $J$ Immunol 172:6087-6092. 
6) Binder, R., D. Han, and P. Srivastava. 2000. CD91: a receptor for heat shock protein gp96. Nature Immunol. 1:151-155.

7) Delneste, Y., G. Magistrelli, J. Gauchat, J. Haeuw, J. Aubry, K. Nakamura, N. Kawakami-Honda, L. Goetsch, T. Sawamura, J. Bonnefoy, and P. Jeannin. 2002. Involvement of LOX-1 in dendritic cell-mediated antigen cross-presentation. Immunity 17:353-362.

8) Becker, T., F.U. Hartl, and F. Wieland. 2002. CD40, an extracellular receptor for binding and uptake of Hsp70-peptide complexes. J Cell Biol 158:1277-1285.

9) Berwin, B., J.P. Hart, S. Rice, C. Gass, S.V. Pizzo, S.R. Post, and C.V. Nicchitta. 2003. Scavenger receptor-A mediates gp96/GRP94 and calreticulin internalization by antigen-presenting cells. Embo J 22:6127-6136.

10) SenGupta, D., P.J. Norris, T.J. Suscovich, M. Hassan-Zahraee, H.F. Moffett, A. Trocha, R. Draenert, P.J. Goulder, R.J. Binder, D.L. Levey, B.D. Walker, P.K. Srivastava, and C. Brander. 2004. Heat shock protein-mediated cross-presentation of exogenous HIV antigen on HLA class I and class II. J Immunol 173:1987-1993.

11) Haug, M., L. Dannecker, C.P. Schepp, W.W. Kwok, D. Wernet, J.H. Buckner, H. Kalbacher, G.E. Dannecker, and U. Holzer. 2005. The heat shock protein Hsp70 enhances antigen-specific proliferation of human $\mathrm{CD}^{+}$memory T cells. Eur J Immunol 35:3163-3172.

12) Ramirez, S.R., H. Singh-Jasuja, T. Warger, S. Braedel-Ruoff, N. Hilf, K. Wiemann, H.G. Rammensee, and H. Schild. 2005. Glycoprotein 96-activated dendritic cells induce a CD8-biased T cell response. Cell Stress Chaperones 10:221-229.

13) Udono, H., and P.K. Srivastava. 1994. Comparison of tumor-specific immunogenicities of stress-induced proteins gp96, hsp90, and hsp70. J Immunol 152:5398-5403.

14) Kunisawa, J., and N. Shastri. 2006. Hsp90alpha chaperones large C-terminally extended proteolytic intermediates in the MHC class I antigen processing pathway. Immunity 24:523-534.

15) Basu, S., R.J. Binder, R. Suto, K.M. Anderson, and P.K. Srivastava. 2000. Necrotic but not apoptotic cell death releases heat shock proteins, which deliver a partial maturation signal to dendritic cells and activate the NF-kappa B pathway. Int Immunol 12:1539-1546.

16) Asea, A., M. Rehli, E. Kabingu, J.A. Boch, O. Bare, P.E. Auron, M.A. Stevenson, and S.K. Calderwood. 2002. Novel signal transduction pathway utilized by extracellular HSP70: role of toll-like receptor (TLR) 2 and TLR4. J Biol Chem 277:15028-15034.

17) Vabulas, R.M., P. Ahmad-Nejad, S. Ghose, C.J. Kirschning, R.D. Issels, and H. Wagner. 2002. HSP70 as endogenous stimulus of the Toll/interleukin-1 receptor signal pathway. J Biol Chem 277:15107-15112.

18) Basu, S., R.J. Binder, T. Ramalingam, and P.K. Srivastava. 2001. CD91 is a common receptor for heat shock proteins gp96, hsp90, hsp70, and calreticulin. Immunity 14:303-313.

19) Becker, T., F.U. Hartl, and F. Wieland. 2002. CD40, an extracellular receptor for binding and uptake of Hsp70-peptide complexes. J Cell Biol 158:1277-1285.

20) Rodriguez, A., A. Regnault, M. Kleijmeer, P. Ricciardi-Castagnoli, and S. Amigorena. 1999. Selective transport of internalized antigens to the cytosol for MHC class I presentation in dendritic cells. Nat Cell Biol 1:362-368.
21) Hotta, C., H. Fujimaki, M. Yoshinari, M. Nakazawa, and M. Minami. 2006. The delivery of an antigen from the endocytic compartment into the cytosol for cross-presentation is restricted to early immature dendritic cells. Immunology 117:97-107.

22) Guermonprez, P., L. Saveanu, M. Kleijmeer, J. Davoust, P. Van Endert, and S. Amigorena. 2003. ER-phagosome fusion defines an MHC class I cross-presentation compartment in dendritic cells. Nature 425:397-402.

23) Houde, M., S. Bertholet, E. Gagnon, S. Brunet, G. Goyette, A. Laplante, M.F. Princiotta, P. Thibault, D. Sacks, and M. Desjardins. 2003. Phagosomes are competent organelles for antigen crosspresentation. Nature 425:402-406.

24) Ackerman, A.L., C. Kyritsis, R. Tampe, and P. Cresswell. 2003. Early phagosomes in dendritic cells form a cellular compartment sufficient for cross presentation of exogenous antigens. Proc Natl Acad Sci U S A 100:12889-12894.

25) Ackerman, A.L., C. Kyritsis, R. Tampe, and P. Cresswell. 2005. Access of soluble antigens to the endoplasmic reticulum can explain cross-presentation by dendritic cells. Nat Immunol 6:107-113.

26) Ackerman, A.L., A. Giodini, and P. Cresswell. 2006. A role for the endoplasmic reticulum protein retrotranslocation machinery during crosspresentation by dendritic cells. Immunity 25:607-617.

27) Gromme, M., F.G. Uytdehaag, H. Janssen, J. Calafat, R.S. van Binnendijk, M.J. Kenter, A. Tulp, D. Verwoerd, and J. Neefjes. 1999. Recycling MHC class I molecules and endosomal peptide loading. Proc Natl Acad Sci U S A 96:10326-10331.

28) Shen, L., L.J. Sigal, M. Boes, and K.L. Rock. 2004. Important role of cathepsin $\mathrm{S}$ in generating peptides for TAP-independent MHC class I crosspresentation in vivo. Immunity 21:155-165.

29) Suto, R., and P.K. Srivastava. 1995. A mechanism for the specific immunogenicity of heat shock protein-chaperoned peptides. Science 269:1585-1588.

30) Castellino, F., P.E. Boucher, K. Eichelberg, M. Mayhew, J.E. Rothman, A.N. Houghton, and R.N. Germain. 2000. Receptormediated uptake of antigen/heat shock protein complexes results in major histocompatibility complex class I antigen presentation via two distinct processing pathways. J Exp Med 191:1957-1964.

31) Moroi, Y., M. Mayhew, J. Trcka, M.H. Hoe, Y. Takechi, F.U. Hartl, J.E. Rothman, and A.N. Houghton. 2000. Induction of cellular immunity by immunization with novel hybrid peptides complexed to heat shock protein 70. Proc Natl Acad Sci U S A 97:3485-3490.

32) Kurotaki, T., Y. Tamura, G. Ueda, J. Oura, G. Kutomi, Y. Hirohashi, H. Sahara, T. Torigoe, H. Hiratsuka, H. Sunakawa, K. Hirata, and N. Sato. 2007. Efficient Cross-Presentation by Heat Shock Protein 90-Peptide Complex-Loaded Dendritic Cells via an Endosomal Pathway. J Immunol 179:1803-1813.

33) Accapezzato, D., V. Visco, V. Francavilla, C. Molette, T. Donato, M. Paroli, M.U. Mondelli, M. Doria, M.R. Torrisi, and V. Barnaba. 2005. Chloroquine enhances human $\mathrm{CD}^{+} \mathrm{T}$ cell responses against soluble antigens in vivo. J Exp Med 202:817-828. 